\title{
Senkt Progesteron die Anfallsfrequenz?
}

Fragestellung: Hat Progesteron eine Wirkung bei Frauen mit therapierefraktärer Epilepsie mit fokalen Anfällen?

Hintergrund: Da bei Frauen Anfälle häufig mit der Menstruation oder in Clustern auftreten, ist es naheliegend, die Rolle von reproduktiven Steroiden und deren mögliche Wirkung auf Epilepsie zu untersuchen. Der Begriff von Anfällen, die sich periodengebunden als sogenannte katameniale Epilepsie manifestieren, stützt sich auf die Annahme, dass Hormone neuroaktive Eigenschaften haben und ihr Spiegel über den Menstruationszyklus eine rhythmische Schwankung hat. Auf zellulärer Ebene hat das Gehirn zum Teil strukturelle und elektrophysiologische Antwortmuster auf diese Steroide.

Patientinnen und Methodik: Die Studie umfasste Frauen im Alter von 13-45 Jahren mit therapierefraktären Anfällen, die resistent auf mehr als zwei antiepileptische Standardmedikationen waren, einen im EEG dokumentierten fokalen Anfallsbeginn und mehr als zwei Anfälle im Monat in den drei Monaten vor Studienaufnahme.

Menstruationszyklusintervalle waren 23 bis 35 Tage. Frauen mit fortschreitend neurologischen Erkrankungen oder Systemerkrankungen wurden ausgeschlossen. Die Studienteilnehmerinnen wurden nach drei Baseline-Menstruationszyklen in katemanial und nicht-katamenial stratifiziert und 2:1 zu Progesteron oder Placebo randomisiert. Die Verumgruppe erhielt dreimal täglich Progesteron $200 \mathrm{mg}$ an den Tagen 14 bis 28 des Behandlungszyklus.

Primärer Studienendpunkt war der prozentuale Anteil von Respondern für alle Anfälle, sekundärer Endpunkt eine 50 \%ige Reduktion in durchschnittlicher täglicher Anfallsfrequenz.

Ergebnisse: Von insgesamt 294 randomisierten Frauen wurden 164 in den nicht-katamenialen und 30 in den katamenialen Arm aufgenommen. In der Baseline-Phase erwiesen sich 168 (36,4\%) als Drop-outs wegen inadäquater Länge des Menstruationszyklus. Bezogen auf den primären Endpunkt der Anfallsfrequenz zeigte sich keine signifikante Differenz im Anteil von Respondern für alle Anfallsformen kombiniert, zwischen Progesteron- und Placeboarm in der katamenialen und nicht-katamenialen Gruppe. Auch gab es kein unterschiedliches Ansprechen bezogen auf unterschiedliche Anfallstypen.

Bei Anfallsfrequenz zeigte sich kein signifikanter Unterschied zwischen Progesteron und Placebo, weder im Hinblick auf proportionelle Veränderungen der Anfallsfrequenz insgesamt oder unterschiedliche Anfallssubtypen. Serumprogesteronspiegel war in der Behandlungsphase

Herzog AG, Fowler KM, Smithson SD et al. Progesterone vs placebo therapy for women with epilepsy: A randomized clinical trial. Neurology 2012; 78; 1959-66 signifikant höher, aber es bestanden keine Unterschiede bei den Antiepileptikaspiegeln.

In einer präspezifizierten sekundären Subanalyse zeigte sich, dass das Ausmaß der perimenstruellen Anfallsexazerbation ein signifikanter Prädiktor für Response gegenüber Progesteron, aber nicht für Placebo war. Mit zunehmendem Level der perimenstuellen Anfallsexazerbation stieg die Responderrate von $21 \%$ auf $57 \%$ unter Progesteron und blieb bei 19-20\% unter Placebo. Parallel hierzu ging die Anfallsfrequenz unter Progesteron stark zurück, während sich unter Placebo kein Unterschied zeigte.

Schlussfolgerungen: Die Autoren schließen, dass im Hinblick auf den primären Endpunkt zwar kein Unterschied zwischen katamenialer und nicht-katamenialer Gruppe besteht. Das Ausmaß der perimenstruellen Anfallsexazerbation kann jedoch als ein signifikanter Prädiktor der Responderrate auf Progesteron gewertet werden. Für eine Subgruppe von Frauen mit perimenstrueller Anfallsexazerbation hat Progesteron einen klinisch relevanten Nutzen.

\section{- Kommentar von Dr. Oliver Kastrup In Einzelfällen sinnvoll}

Diese methodologisch sehr gut aufgebaute Studie widmet sich der klinisch attraktiven Fragestellung, ob nicht bei Frauen mit therapierefraktären fokalen Anfällen gegenüber Standardantiepileptika eine Zugabe von Progesteron die Anfallsfrequenz vermindern kann. Obwohl das ein verlockendes Konzept wäre, belegt die Studie leider, dass zyklisch verabreichtes Progesteron bei Frauen mit therapierefraktärer fokaler Epilepsie generell nicht effektiv ist. Das gilt sowohl bei rein katamenialen als auch nicht-katamenial gebundenen Anfällen. Da allerdings eine Post-hoc-Analyse eine Subpopulation der Frauen mit hoher Zahl an perimenstruell exazerbierenden Anfällen als aufsteigend responsiv gegenüber Progesteron zeigte, könnte dieses eine für diese Frauen zumindest sehr hilfreiche und tolerable Therapieoption darstellen, um eine perimenstruell hohe Anfallsfrequenz zu reduzieren. Ein Therapieversuch mit Progesteron 200 mg dreimal täglich für 14 Tage perimenstruell könnte Patientinnen mit dieser Anfallsform gegebenenfalls additiv angeboten werden.

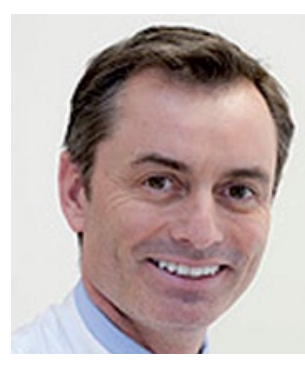

Dr. med. Oliver Kastrup, Essen

Leitender Oberarzt der Klinik für Neurologie, Universitätsklinikum Essen E-Mail: oliver.kastrup@uni-due.de 